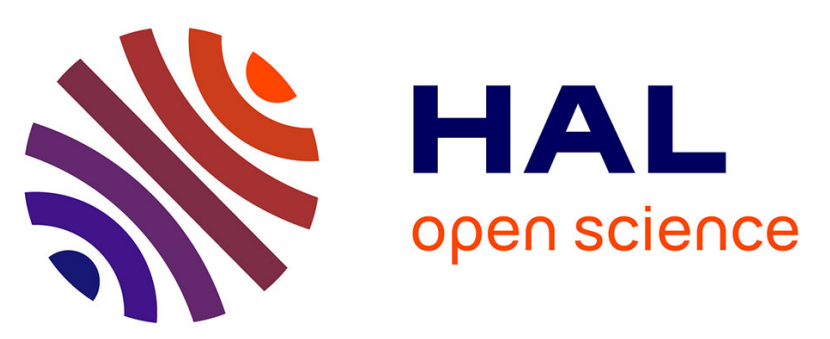

\title{
Status of LUMINEU program to search for neutrinoless double beta decay of 100Mo with cryogenic $\mathrm{ZnMoO} 4$ scintillating bolometers
}

F. A. Danevich, L. Bergé, R. S. Boiko, M. Chapellier, D. M. Chernyak, N. Coron, L. Devoyon, A.-A. Drillien, L. Dumoulin, C. Enss, et al.

\section{To cite this version:}

F. A. Danevich, L. Bergé, R. S. Boiko, M. Chapellier, D. M. Chernyak, et al.. Status of LUMINEU program to search for neutrinoless double beta decay of 100Mo with cryogenic ZnMoO4 scintillating bolometers. Matrix Elements for the Double-beta-decay EXperiments (MEDEX'15), Jun 2015, Prague, Czech Republic. pp.020007, 10.1063/1.4934896 . in2p3-01226305

\section{HAL Id: in2p3-01226305 https://hal.in2p3.fr/in2p3-01226305}

Submitted on 24 Mar 2020

HAL is a multi-disciplinary open access archive for the deposit and dissemination of scientific research documents, whether they are published or not. The documents may come from teaching and research institutions in France or abroad, or from public or private research centers.
L'archive ouverte pluridisciplinaire HAL, est destinée au dépôt et à la diffusion de documents scientifiques de niveau recherche, publiés ou non, émanant des établissements d'enseignement et de recherche français ou étrangers, des laboratoires publics ou privés. 


\title{
Status of LUMINEU program to search for neutrinoless double beta decay of ${ }^{100} \mathrm{Mo}$ with cryogenic $\mathrm{ZnMoO}_{4}$ scintillating bolometers
}

\author{
F.A. Danevich ${ }^{1, a), b)}$, L. Bergé2 ${ }^{2}$ R.S. Boiko ${ }^{1}$, M. Chapellier ${ }^{2}$, D.M. Chernyak ${ }^{1}$, \\ N. Coron $^{3}$, L. Devoyon ${ }^{4}$, A.-A. Drillien ${ }^{2}$, L. Dumoulin ${ }^{2}$, C. Enss $^{5}$,

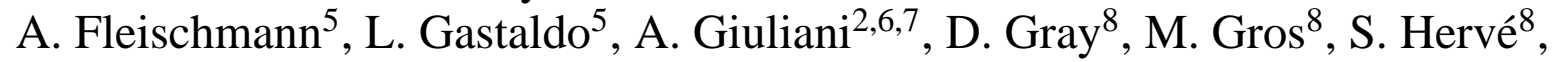 \\ V. Humbert ${ }^{2}$, I.M. Ivanov ${ }^{9}$, A. Juillard ${ }^{10}$, V.V. Kobychev ${ }^{1}$, F. Koskas ${ }^{4}$, M. Loidl ${ }^{11}$, \\ P. Magnier ${ }^{8}$, E.P. Makarov ${ }^{9}$, M. Mancuso ${ }^{2,6}$, P. de Marcillac ${ }^{2}$, S. Marnieros ${ }^{2}$, \\ C. Marrache-Kikuchi ${ }^{2}$, X.-F. Navick ${ }^{8}$, C. Nones ${ }^{8}$, E. Olivieri ${ }^{2}$, B. Paul ${ }^{8}$, \\ Y. Penichot ${ }^{8}$, G. Pessina ${ }^{7,12}$, O. Plantevin ${ }^{2}$, D.V. Poda ${ }^{1,2}$, T. Redon ${ }^{3}$, \\ M. Rodrigues ${ }^{11}$, V.N. Shlegel ${ }^{9}$, O. Strazzer ${ }^{4}$, M. Tenconi ${ }^{2}$, L. Torres ${ }^{3}$, \\ V.I. Tretyak ${ }^{1,13}$, Ya.V. Vasiliev ${ }^{9}$, M. Velazquez ${ }^{14}$ and O. Viraphong ${ }^{14}$ \\ ${ }^{1}$ Institute for Nuclear Research, MSP 03680 Kyiv, Ukraine \\ ${ }^{2}$ Centre de Sciences Nucléaires et de Sciences de la Matière, CNRS/IN2P3, Université Paris-Sud, 91405 Orsay, \\ France \\ ${ }^{3}$ IAS, CNRS, Université Paris-Sud, 91405 Orsay, France \\ ${ }^{4}$ CEA, Centre d'Etudes Saclay, Orphée, 91191 Gif-Sur-Yvette Cedex, France \\ ${ }^{5}$ Kirchhoff Institute for Physics, Heidelberg University, D-69120 Heidelberg, Germany \\ ${ }^{6}$ Dipartimento di Scienza e Alta Tecnologia dell'Università dell'Insubria, 22100 Como, Italy \\ ${ }^{7}$ INFN, Sezione di Milano-Bicocca, 20126 Milano, Italy \\ ${ }^{8}$ CEA, Centre d'Etudes Saclay, IRFU, 91191 Gif-Sur-Yvette Cedex, France \\ ${ }^{9}$ Nikolaev Institute of Inorganic Chemistry, 630090 Novosibirsk, Russia \\ ${ }^{10}$ IPNL, Université de Lyon, Université Lyon 1, CNRS/IN2P3, 69622 Villeurbanne cedex, France \\ ${ }^{11}$ CEA, LIST, LNHB, 91191 Gif-Sur-Yvette Cedex, France \\ ${ }^{12}$ Dipartimento di Fisica dell'Universitá di Milano-Bicocca, 20126 Milano, Italy \\ ${ }^{13}$ INFN, sezione di Roma "La Sapienza", I-00185 Rome, Italy \\ ${ }^{14}$ ICMCB, CNRS, Université de Bordeaux, 33608 Pessac Cedex, France \\ a)Corresponding author: danevich@kinr.kiev.ua \\ b)URL: http://lpd.kinr.kiev.ua/
}

\begin{abstract}
The LUMINEU program aims at performing a pilot experiment on $0 v 2 \beta$ decay of ${ }^{100}$ Mo using radiopure $\mathrm{ZnMoO}_{4}$ crystals enriched in ${ }^{100} \mathrm{Mo}$ operated as cryogenic scintillating bolometers. Large volume $\mathrm{ZnMoO}_{4}$ crystal scintillators $(\sim 0.3 \mathrm{~kg})$ were developed and tested showing high performance in terms of radiopurity, energy resolution and $\alpha / \beta$ particle discrimination capability. Zinc molybdate crystal scintillators enriched in ${ }^{100} \mathrm{Mo}$ were grown for the first time by the low-thermal-gradient Czochralski technique with a high crystal yield and an acceptable level of enriched molybdenum irrecoverable losses. A background level of $\sim 0.5$ counts/(yr keV ton) in the region of interest can be reached in a large detector array thanks to the excellent detectors radiopurity and particle discrimination capability, suppression of randomly coinciding events by pulse-shape analysis, and anticoincidence cut. These results pave the way to future sensitive searches based on the LUMINEU technology, capable of approaching and exploring the inverted hierarchy region of the neutrino mass pattern.
\end{abstract}




\section{INTRODUCTION}

Search for neutrinoless double beta $(0 v 2 \beta)$ decay is considered as the only experimentally viable approach to test the Majorana nature of neutrino and the lepton number conservation. The $0 v 2 \beta$ decay, if mediated by the mass mechanism, is able to measure the effective Majorana neutrino mass and to determine the hierarchy of neutrino masses. In addition, the process can be mediated by right handed currents in weak interactions, existence of massless (or very light) Nambu-Goldstone bosons (majorons), and other effects beyond the Standard Model [1, 2, 3, 4, 5]. The isotope ${ }^{100} \mathrm{Mo}$ is one of the most promising nuclei to search for $0 v 2 \beta$ decay thanks to the high energy of the decay $\left(Q_{2 \beta}=3034.40(17) \mathrm{keV}[6]\right)$, comparatively high natural isotopic abundance $(\delta=9.82(31) \%$ [7]) and the favorable theoretical predictions (see, e.g., [1,2] and references therein). Here we report a status of the LUMINEU program (Luminescent Underground Molybdenum Investigation for NEUtrino mass and nature) which aims at preparing and construction of a next-generation $0 v 2 \beta$ decay experiment capable to explore the inverted hierarchy region of neutrino mass with cryogenic scintillating bolometers based on radiopure $\mathrm{ZnMoO}_{4}$ crystals enriched in ${ }^{100} \mathrm{Mo}$ [8].

\section{DETECTORS DEVELOPMENT}

Properties of $\mathrm{ZnMoO}_{4}$ crystal as cryogenic low temperature scintillator were checked for the first time in [9]. Large volume high optical quality $\mathrm{ZnMoO}_{4}$ crystal scintillators from deeply purified molybdenum with mass larger than $0.3 \mathrm{~kg}$ were then developed in the framework of the LUMINEU project and their optical, luminescent, diamagnetic, thermal and bolometric properties were tested [10]. Cryogenic scintillating bolometers based on $\mathrm{ZnMoO}_{4} \mathrm{crystals}$ show high performance in terms of energy resolution (e.g., FWHM $=6 \mathrm{keV}$ at $2615 \mathrm{keV}$ was reached in [11]), powerful $\alpha / \beta$ particle discrimination capability (on the level of $15 \sigma$ ) and a very low level of radioactive contamination [12]. The data on radioactive contamination of a LUMINEU $\mathrm{ZnMoO}_{4}$ crystal scintillator with mass $334 \mathrm{~g}$ are presented in Table 1 . The level of thorium and radium contamination fully satisfies the LUMINEU requirements.

TABLE 1. Radioactive contamination of LUMINEU $\mathrm{ZnMoO}_{4}$ crystal scintillator [12].

\begin{tabular}{lll}
\hline Chain & Nuclide (sub-chain) & Activity $(\mu \mathbf{B q} / \mathbf{k g})$ \\
\hline${ }^{232} \mathrm{Th}$ & ${ }^{190} \mathrm{Pt}$ & $3.8 \pm 1.2$ \\
& ${ }^{232} \mathrm{Th}$ & $\leq 2.3$ \\
& ${ }^{228} \mathrm{Th}$ & $\leq 5.3$ \\
${ }^{235} \mathrm{U}$ & ${ }^{235} \mathrm{U}$ & $\leq 3.3$ \\
${ }^{238} \mathrm{U}$ & ${ }^{238} \mathrm{U}$ & $\leq 1.8$ \\
& ${ }^{226} \mathrm{Ra}$ & $\leq 4.8$ \\
& ${ }^{210} \mathrm{Po}$ & $1271 \pm 22$ \\
\hline
\end{tabular}

$\mathrm{A} \mathrm{Zn}^{100} \mathrm{MoO}_{4}$ crystal with a mass of $0.17 \mathrm{~kg}$ was grown from molybdenum enriched in ${ }^{100} \mathrm{Mo}$ [13] as an important step of the project. The output of the crystal boule was $84 \%$, while the irrecoverable losses of enriched molybdenum were found to be $\approx 4 \%$ in the total production cycle, which demonstrates an important advantage of the purification method and of the low-thermal-gradient Czochralski technique used for the crystal growing. Recently, a large enriched $\mathrm{Zn}^{100} \mathrm{MoO}_{4}$ crystal boule with mass $\approx 1.4 \mathrm{~kg}$ was grown (see Fig. 1) and two detectors were produced. Test of the detectors performance and radioactive contamination is in progress.

\section{BACKGROUND CONDITIONS IN EXPERIMENT WITH $\mathrm{Zn}^{100} \mathrm{MoO}_{4}$ DETECTORS $^{-1}$}

To estimate background in conditions of an experiment with $\mathrm{Zn}^{100} \mathrm{MoO}_{4}$ scintillating bolometers, a Monte Carlo simulation of $48 \mathrm{Zn}^{100} \mathrm{MoO}_{4}$ detectors (with a mass $495 \mathrm{~g}$, size $\oslash 60 \times 40 \mathrm{~mm}$ each) in the EDELWEISS set-up is in progress (some preliminary results can be found in [14]). We simulated contamination of $\mathrm{Zn}^{100} \mathrm{MoO}_{4}$ crystals and the nearest to the detectors materials (copper holders, polyethylene terephthalate (PTFE) clamps, reflective foil) by ${ }^{238} \mathrm{U}$ and ${ }^{232} \mathrm{Th}$ daughters. We have also simulated cosmogenic nuclides in the $\mathrm{Zn}^{100} \mathrm{MoO}_{4}$ crystals and the copper holder. The results of the simulation are summarized in Table 2, which reports also the assumed contaminations. A rejection factor of $99.9 \%$ was applied every time when a $\beta$ event is emitted in sequence with an $\alpha$ event within the 


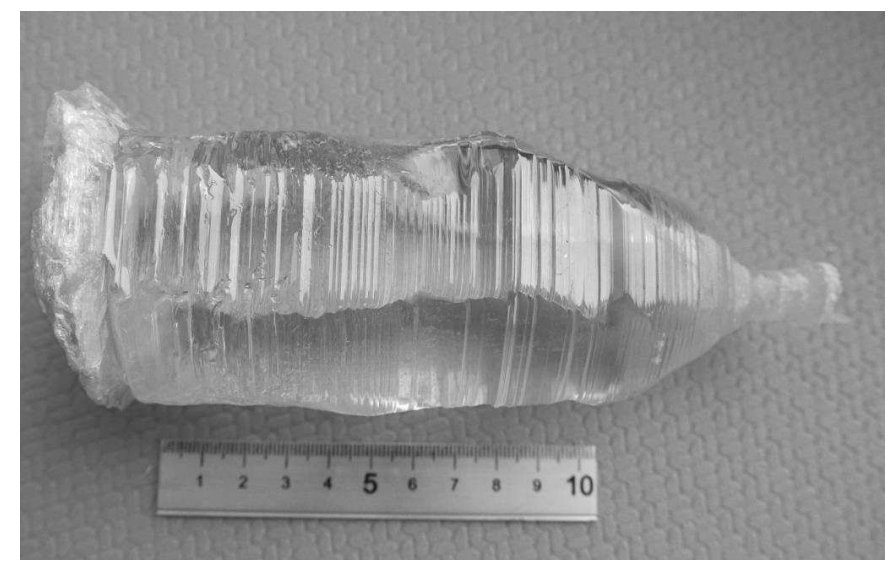

FIGURE 1. Boule of $\mathrm{Zn}^{100} \mathrm{MoO}_{4}$ crystal with mass $\approx 1.4 \mathrm{~kg}$ produced from molybdenum isotopically enriched in ${ }^{100} \mathrm{Mo}$.

time resolution of the bolometer. We also assumed suppression of the bulk and surface ${ }^{208} \mathrm{Tl}\left({ }^{228} \mathrm{Th}\right.$ chain) by a factor $10^{3}$ applying the delayed anti-coincidence with a prompt $\alpha$ event from ${ }^{212} \mathrm{Bi}$ [15]. Finally, anticoincidence rejection of background thanks to the multi-detector structure was taken into account. The simulated background energy spectrum and its main components are shown in Fig. 2.

TABLE 2. Monte Carlo simulated background contributions in an experiment with $\mathrm{Zn}^{100} \mathrm{MoO}_{4}$ scintillating bolometers installed in the EDELWEISS set-up.

\begin{tabular}{lll}
\hline Source of background & Activity $(\mu \mathbf{B q} / \mathbf{k g})$ & Background, counts/(yr keV kg) \\
\hline${ }^{228} \mathrm{Th}$ in $\mathrm{Zn}^{100} \mathrm{MoO}_{4}$ crystals & 10 & $8.6 \times 10^{-6}$ \\
${ }^{226} \mathrm{Ra}$ in $\mathrm{Zn}^{100} \mathrm{MoO}_{4}$ crystals & 10 & $3.1 \times 10^{-8}$ \\
${ }^{88} \mathrm{Y}$ in $\mathrm{Zn}^{100} \mathrm{MoO}_{4}$ crystals & 0.3 & $6.3 \times 10^{-7}$ \\
${ }^{56} \mathrm{Co}$ in $\mathrm{Zn}^{100} \mathrm{MoO}_{4}$ crystals & 0.06 & $6.2 \times 10^{-5}$ \\
${ }^{232} \mathrm{Th}$ in $\mathrm{Zn}^{100} \mathrm{MoO}_{4}$ surface layer & 0.5 & $1.2 \times 10^{-5}$ \\
${ }^{238} \mathrm{U}$ in $\mathrm{Zn}^{100} \mathrm{MoO}_{4}$ surface layer & 2.4 & $1.5 \times 10^{-4}$ \\
${ }^{228} \mathrm{Th}$ in copper holders & 20 & $1.3 \times 10^{-6}$ \\
${ }^{226} \mathrm{Ra}$ in copper holders & 70 & $1.5 \times 10^{-7}$ \\
${ }^{56} \mathrm{Cu}$ in copper holders & 0.2 & $6.6 \times 10^{-5}$ \\
${ }^{228} \mathrm{Th}$ in PTFE clamps & 100 & $9.6 \times 10^{-6}$ \\
${ }^{226} \mathrm{Ra}$ in PTFE clamps & 60 & $7.5 \times 10^{-7}$ \\
${ }^{228} \mathrm{Th}$ in reflective foil & 100 & $7.5 \times 10^{-5}$ \\
${ }^{226} \mathrm{Ra}$ in reflective foil & 60 & $2.1 \times 10^{-5}$ \\
$\mathrm{Random}$ coincidence of ${ }^{100} \mathrm{Mo} 2 v 2 \beta$ events & 8130 & $\approx 10^{-4}$ \\
Total & & $\approx 5 \times 10^{-4}$ \\
\hline
\end{tabular}

As it was pointed out in [16] random coincidence of $2 v 2 \beta$ events can be the main background in bolometric $0 v 2 \beta$ experiments with ${ }^{100}$ Mo due to the poor time resolution of cryogenic detectors. However, the background can be reduced to the level of $\approx 10^{-4}$ counts/(yr keV kg) with the help of pulse-shape discrimination [17]. Therefore, a total background counting rate $\approx 5 \times 10^{-4}$ counts/(yr keV kg) can be reached, which corresponds to 3 counts/(yr ton) in the region of interest (assuming $6 \mathrm{keV}$ window centered at the $0 v 2 \beta$ peak position).

\section{CONCLUSIONS}

Cryogenic scintillating bolometers based on large volume $(\sim 0.3 \mathrm{~kg}) \mathrm{ZnMoO}_{4}$ crystals, developed in the framework of the LUMINEU project, show excellent performance: a few keV energy resolution, 15 sigma alpha/beta particle discrimination power at the $Q_{2 \beta}$ value of ${ }^{100} \mathrm{Mo}$. Radioactive contamination of $\mathrm{ZnMoO}_{4}$ crystal scintillators is very 


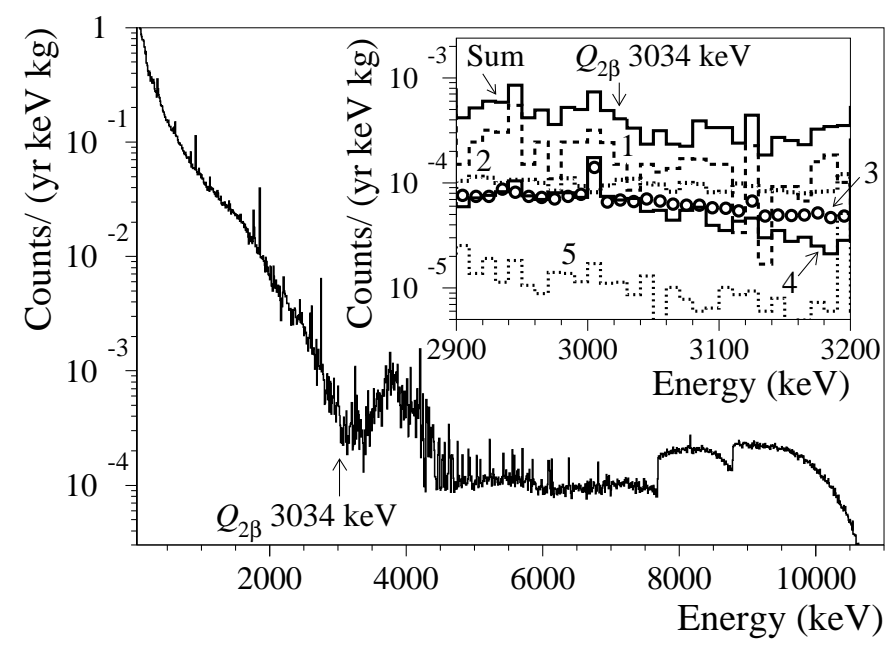

FIGURE 2. Energy spectra of $48 \mathrm{Zn}^{100} \mathrm{MoO}_{4}$ detectors in EDELWEISS set-up simulated with the help of GEANT4 package. (Inset) The main components of background are shown: (1) surface contamination of $\mathrm{Zn}^{100} \mathrm{MoO}_{4}$ crystals, (2) radioactive contamination of the reflecting foil surrounding the crystal, (3) bulk contamination of $\mathrm{Zn}^{100} \mathrm{MoO}_{4}$ crystals, contamination of copper holders (4) and PTFE clamps (5).

low thanks to the deep purification of molybdenum: activity of ${ }^{228} \mathrm{Th}$ and ${ }^{226} \mathrm{Ra}$ does not exceed $5 \mu \mathrm{Bq} / \mathrm{kg}$. Enriched $\mathrm{Zn}^{100} \mathrm{MoO}_{4}$ crystals with a mass of $0.17 \mathrm{~kg}$ and $1.4 \mathrm{~kg}$ were grown for the first time by the low-thermal-gradient Czochralski technique. The production cycle provided a high yield of the crystal boule (more than $80 \%$ of the initial charge) and an acceptable level of irrecoverable losses of molybdenum $(\approx 4 \%)$. A background level of $\sim 0.5$ counts/(yr keV ton) in the region of interest can be reached in a large detector array thanks to the excellent detectors radiopurity and particle discrimination capability, suppression of randomly coinciding events by pulse-shape analysis, and anticoincidence cut. It should be stressed that also $\mathrm{Li}_{2} \mathrm{MoO}_{4}$ cryogenic scintillating bolometers were developed and tested as promising detectors for a high sensitivity experiment with ${ }^{100} \mathrm{Mo}$ [18]. These results pave the way to future sensitive searches based on the LUMINEU technology, capable of exploring the inverted hierarchy region of the neutrino mass pattern. The LUMINEU activity is part of the CUPID project $[19,20]$, a proposed bolometric tonnescale experiment to be built as a follow-up to CUORE and exploiting as much as possible the CUORE infrastructures.

\section{REFERENCES}

[1] J.D. Vergados, H. Ejiri and F. Šimkovic, Rep. Prog. Phys. 75, 106301 (2012).

[2] J. Barea, J. Kotila, and F. Iachello, Phys. Rev. Lett. 109, 042501 (2012).

[3] W. Rodejohann, J. Phys. G: Nucl. Part. Phys. 39, 124008 (2012).

[4] F.F. Deppisch, M. Hirsch and H. Päs, J. Phys. G: Nucl. Part. Phys. 39, 124007 (2012).

[5] S.M. Bilenky, C. Giunti, Int. J Mod. Phys. A 30, 1530001 (2015).

[6] S. Rahaman et al., Phys. Lett. B 662, 111 (2008).

[7] M. Berglund and M.E. Wieser, Pure Appl. Chem. 83, 397 (2011).

[8] M. Tenconi, for the LUMINEU collaboration, Phys. Proc. 61, 782 (2015).

[9] L. Gironi et al., JINST 5, P11007 (2010).

[10] L. Bergé et al., JINST 9, P06004 (2014).

[11] J.W. Beeman et al., Eur. Phys. J. C 72, 2142 (2012).

[12] E. Armengaud et al., JINST 10, P05007 (2015).

[13] A.S. Barabash et al., Eur. Phys. J. C 74, 3133 (2014).

[14] D. Chernyak, PhD thesis, arXiv:1507.04591v1 [physics.ins-det].

[15] J.W. Beeman et al., Phys. Lett. B 710, 318 (2012).

[16] D.M. Chernyak et al., Eur. Phys. J. C 72, 1989 (2012).

[17] D.M. Chernyak et al., Eur. Phys. J. C 74, 2913 (2014).

[18] T.B. Bekker et al., Astropart. Phys. 72, 38 (2016).

[19] G. Wang et al., arXiv:1504.03599v1 [physics.ins-det].

[20] G. Wang et al., arXiv:1504.03612v1 [physics.ins-det]. 